\title{
Global color estimation of special-effect coatings from measurements by commercially available portable multiangle spectrophotometers
}

\author{
A. Ferrero, ${ }^{1, *}$ J. Campos, ${ }^{1}$ E. Perales, ${ }^{2}$ F. M. Martínez-Verdú, ${ }^{2}$ I. van der Lans, ${ }^{3}$ and E. Kirchner ${ }^{3}$ \\ ${ }^{1}$ Instituto de Óptica, Agencia Estatal Consejo Superior de Investigaciones Científicas, \\ c/Serrano 144, Madrid 28006, Spain \\ ${ }^{2}$ Department of Optics, Pharmacology and Anatomy, University of Alicante, \\ Carretera de San Vicente del Raspeig s/n 03690, Alicante, Spain \\ ${ }^{3}$ Color Research Department, AkzoNobel Automotive \& Aerospace Coatings, Sassenheim, The Netherlands \\ ${ }^{*}$ Corresponding author: alejandro.ferrero@csic.es
}

Received September 2, 2014; accepted October 29, 2014; posted November 7, 2014 (Doc. ID 220195); published December 3, 2014

Colors of special-effect coatings have strong dependence on illumination/viewing geometry and an appealing appearance. An open question is to ask about the minimum number of measurement geometries required to completely characterize their observed color shift. A recently published principal components analysis (PCA)-based procedure to estimate the color of special-effect coatings at any geometry from measurements at a reduced set of geometries was tested in this work by using the measurement geometries of commercial portable multiangle spectrophotometers X-Rite MA98, Datacolor FX10, and BYK-mac as reduced sets. The performance of the proposed PCA procedure for the color-shift estimation for these commercial geometries has been examined for 15 special-effect coatings. Our results suggest that for rendering the color appearance of 3D objects covered with special-effect coatings, the color accuracy obtained with this procedure may be sufficient. This is the case especially if geometries of X-Rite MA98 or Datacolor FX10 are used. ( 2014 Optical Society of America OCIS codes: $\quad$ (330.1710) Color, measurement; (330.1720) Color vision; (290.1483) BSDF, BRDF, and BTDF. http://dx.doi.org/10.1364/JOSAA.32.000001

\section{INTRODUCTION}

Special-effect coatings $[1,2]$ consist of a transparent medium containing traditional absorption pigments and flake-shaped effect pigments. The special-effect pigments have a selective reflectance due to their layered structure, which gives rise to interference. This produces a strong color dependence on the illumination/viewing geometry and, in consequence, an appealing appearance. This color effect is interesting for the automotive industry [3] and for other applications such as packaging, cosmetics, and security inks. However, the complex way in which special-effect coatings reflect the radiant flux leads to some experimental challenges. To completely characterize the color of these coatings under any illuminant and for any illumination/viewing geometry, the spectral bidirectional reflectance distribution function (BRDF) [ $\underline{4}]$ has to be measured at adequate measurement geometries to provide all information required to determine the variation of the spectral reflectance with respect to the geometry. Some sophisticated instruments to measure the spectral BRDF in an almost unlimited number of in- and out-of-plane geometries have been recently developed [5-14]. However, industrial applications need more compact and fast systems for colorimetric characterization, and commercially available instruments have a limited number of measurement geometries, following guidelines from some international standards (ASTM, DIN, CIE, etc.). The potential of noncommercial and almost unlimited instruments is key to obtaining detailed BRDF measurements of special-effect coatings in order to obtain a better understanding of these special-effect pigments, coatings, and plastics composed by these instruments and to apply efficiently color quality controls in industry. An open question is to determine the minimum number of measurement geometries required to completely characterize the spectral BRDF of special-effect coatings. The study by Takagi et al. [15] concluded that almost 2000 geometries would be required; however, nowadays this is an unrealizable goal for most industries due to time constraints and cost-efficiency reasons. The ASTM standard for multiangle color measurement of interference pigments [16] recommends eight uniplanar measurement geometries for quality control applications, but it points out that, for other applications, additional geometries are "desirable or needed." Nadal and Early [17] proposed a combination of three illumination angles and five aspecular angles for the adequate colorimetric characterization of these coatings. Ferrero et al. [18] proposed nine geometries resulting from two different illumination angles, one of them combined with five aspecular angles and the other combined with four aspecular angles. This proposal was based on modeling the spectral $\mathrm{BRDF}$ of these coatings as a function of the flake-based parameters $\left(\theta_{\text {inc }}\right.$, or angle of incidence on the interference pigment, and $\theta_{\text {flake }}$, or inclination angle of the flake surface respect to the sample surface) [19] instead of using instrumental spherical geometries defined with respect to the sample surface. This approach assumes that the spectral BRDF is identical within any set of geometries that is defined by a pair of specific values of $\theta_{\text {inc }}$ and $\theta_{\text {flake }}$, and, in turn, every pair of 
flake-based parameters would define a so-called isochromatic line (or isoline). Recently, this assumption has been experimentally proven and published [20].

In the same article by Ferrero et al. [18], a principal components analysis (PCA)-based procedure was presented to estimate the special-effect color at any geometry from the measurements at just the above-mentioned proposed set of nine geometries. These geometries were selected from scratch and do not coincide with those available in the three commercial portable multiangle spectrophotometers (X-Rite MA98, Datacolor FX10, and BYK-mac). This article is intended to quantify and explain the performance of the proposed PCAbased procedure to estimate color of special-effect coatings at any geometry from the set of geometries provided from these instruments, which are widely used in industry. A good performance of this procedure would allow rendering the color of special-effect surfaces from the small number of measurement geometries provided by these commercial portable instruments. In addition, this procedure would allow new methods of color quality controls in many industries to be articulated.

\section{MATERIALS AND METHODS}

\section{A. Description of Special-Effect Samples}

The spectral BRDF of 15 special-effect samples have been measured. Nine of them were prepared by AkzoNobel as part of a more extensive work, including special-effect, metallic, and achromatic coatings, and their color shifts have been already published [21]. Color shifts of the remaining six samples also have been published [22]. Information about the 15 samples is shown in Table 1, including the name of the paint or pigment company, a color description, and the type of pigment (Iriodin, Xirallic, Colorstream, and Meoxal pigments from Merck and Chromaflair pigment from JDSU). As representative examples of the color behavior of these coatings, the color of four of them with different types of pigment (Iriodin, Xirallic, Colorstream, and Chromaflair) are shown in $3 \mathrm{D} \mathrm{L}^{*}-\mathrm{a}^{*}-\mathrm{b}^{*}$ diagrams (Fig. 1) for the first 10 measurement geometries given in Table 2 . As a rule of thumb, the lower the aspecular angles in the table, the higher the values of $\mathrm{L}^{*}$.
The values of two color shift descriptors for these coatings are shown in a bar chart in Fig. 2: Total hue angle variation and chroma variation. The former was defined as "the maximum variation in hue angle along the line with constant aspecular angle $\theta_{\text {asp }}=10^{\circ}$ " and the latter as "the maximum variation of chroma along the line with constant bistatic angle $\theta_{\mathrm{bi}}=10^{\circ}$ " [22]. Here, the aspecular angle refers to the angular deviation between the viewing direction and the specular direction, whereas the bistatic angle is defined as the angle between the viewing direction and the incidence direction. There is an important correlation between the values of $\theta_{\text {asp }}$ and $\theta_{\text {flake }}$, and it is possible to affirm that, in general, the higher the value of $\theta_{\text {asp }}$, the higher the value of $\theta_{\text {flake }}$ of the flakes that specularly reflect the flux toward the viewing direction. This means that, since the orientation distribution is usually peaked at $0^{\circ}$ (flakes and coating are parallel), the reflection of the interference pigments prevails over basecoat scattering at lower values of $\theta_{\text {asp }}$. This is the reason why the measured hue angle varies most within the series of geometries that has a low aspecular angle. Similarly, there is an important correlation between the values of $\theta_{\mathrm{bi}}$ and $\theta_{\text {inc. It }}$ is possible to affirm that, in general, the higher $\theta_{\mathrm{bi}}$, the higher the incidence angle, or $\theta_{\text {inc }}$, at the flakes that specularly reflect the flux toward the viewing direction [22].

The variations of total hue angle and chroma are descriptors that are not necessarily correlated. The 15 studied samples present a wealth of diversity for these values, as can be observed in Fig. 2 .

\section{B. Spectral BRDF Measurements}

GEFE (Gonioespectrofotómetro Español), the goniospectrophotometer developed at the Instituto de Óptica in Agencia Estatal CSIC (IO-CSIC) [5] allows the spectral $\mathrm{BRDF}$ to be measured in the visible range at any geometry, including out-of-plane and retroreflection directions. The spectral BRDF of the special-effect samples have been densely measured by GEFE following a normalized procedure. The measurement geometries result from combining different uniformly spaced values of the spherical coordinates that describe both illumination and detection directions with respect to the sample normal. Polar angles of illumination $\left(\theta_{\mathrm{i}}\right)$ and detection $\left(\theta_{\mathrm{s}}\right)$ varied from $0^{\circ}$ to $70^{\circ}$ in steps of $10^{\circ}$.

Table 1. Special-Effect Coatings Studied in this Work

\begin{tabular}{|c|c|c|c|}
\hline Code Number & Company & Description & Type of Pigment \\
\hline 1 & AkzoNobel Coatings & Green-Blue $^{a}$ & Iriodin \\
\hline 2 & AkzoNobel Coatings & Crystal Silver & Xirallic $\mathrm{Al}_{2} \mathrm{O}_{3} / \mathrm{TiO}_{2}$ \\
\hline 3 & AkzoNobel Coatings & Viola Phantasy & Colorstream \\
\hline 4 & AkzoNobel Coatings & Green Purple & Chromaflair \\
\hline 5 & AkzoNobel Coatings & Green Purple & Chromaflair \\
\hline 6 & AkzoNobel Coatings & Gold Silver & Chromaflair \\
\hline 7 & AkzoNobel Coatings & Gold Silver & Chromaflair \\
\hline 8 & AkzoNobel Coatings & Gold Silver & Chromaflair \\
\hline 9 & Merck & Wahiba Orange & Meoxal \\
\hline 10 & Merck & Lapis Sunlight & Colorstream \\
\hline 11 & Merck & Arctic Fire & Colorstream \\
\hline 12 & Merck & Light Yellow \& Solaris Red & Xirallic $\mathrm{Al}_{2} \mathrm{O}_{3} / \mathrm{TiO}_{2}$ \\
\hline 13 & BASF Coatings & Burgundy \& Olive Green $^{a}$ & Colorstream \\
\hline 14 & BASF Coatings & Laurel Green \& Greenish Blue ${ }^{a}$ & Colorstream \\
\hline 15 & BASF Coatings & Light Blue \& Pale Turquoise ${ }^{a}$ & Colorstream \\
\hline
\end{tabular}



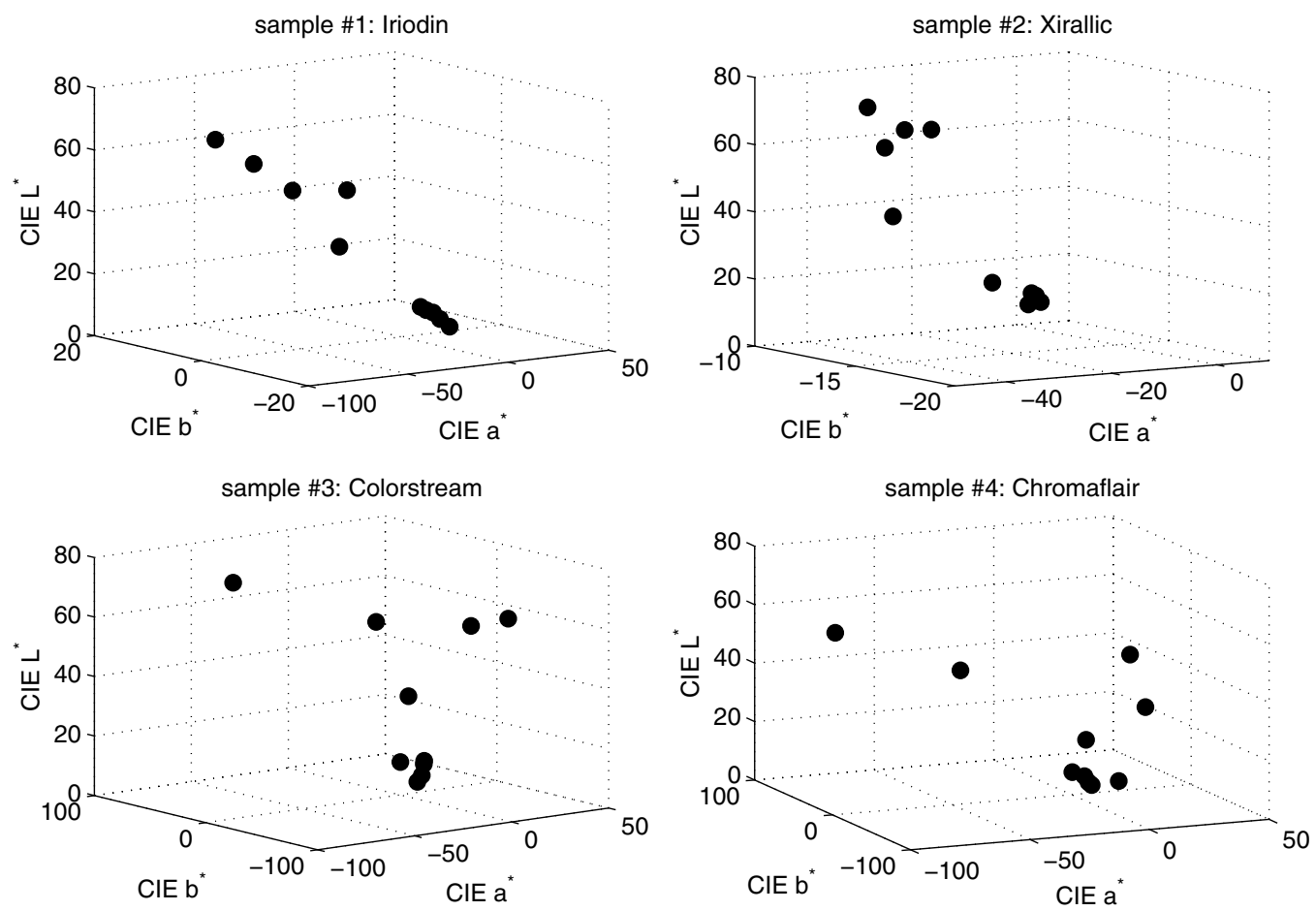

Fig. 1. Representative examples of the color behavior of the studied coatings. The four displayed coatings correspond to different types of pigment (Iriodin, Xirallic, Colorstream, and Chromaflair).

The azimuthal angle of the detection direction $\left(\phi_{\mathrm{s}}\right)$ varied from $0^{\circ}$ to $180^{\circ}$ in steps of $30^{\circ}$, assuming reflection symmetry with respect to the incident plane, whereas the azimuthal angle of the illumination direction $\left(\phi_{\mathrm{i}}\right)$ was fixed, since preliminary measurements showed that anisotropy was negligible under azimuthal rotations of the samples. Therefore, the resulting spectral BRDF is composed of $8 \times 8 \times 7=448$ measurement geometries. However, since those geometries with $\theta_{\mathrm{i}}=0^{\circ}$ or $\theta_{\mathrm{s}}=0^{\circ}$ are identical for every value of $\phi_{\mathrm{s}}$, the number of different geometries is smaller (358).
C. Measurement Geometries in Commercial Instruments The measurement geometries of the commercially available portable multiangle spectrophotometers X-Rite MA98, Datacolor FX10, and BYK-mac are shown in Table 2, together with their corresponding flake-based parameters $\theta_{\text {inc }}$ and $\theta_{\text {flake }}$, calculated as explained in [19]. These geometries are classified in four groups: (1) those common to the four instruments; (2) those common only to X-Rite MA98 and Datacolor FX10; (3) those geometries exclusive for X-Rite MA98; and (4) those exclusive for Datacolor

Table 2. Measurement Geometries Used by Multiangle Spectrophotometers: X-Rite MA98, Datacolor FX10, and BYK-mac

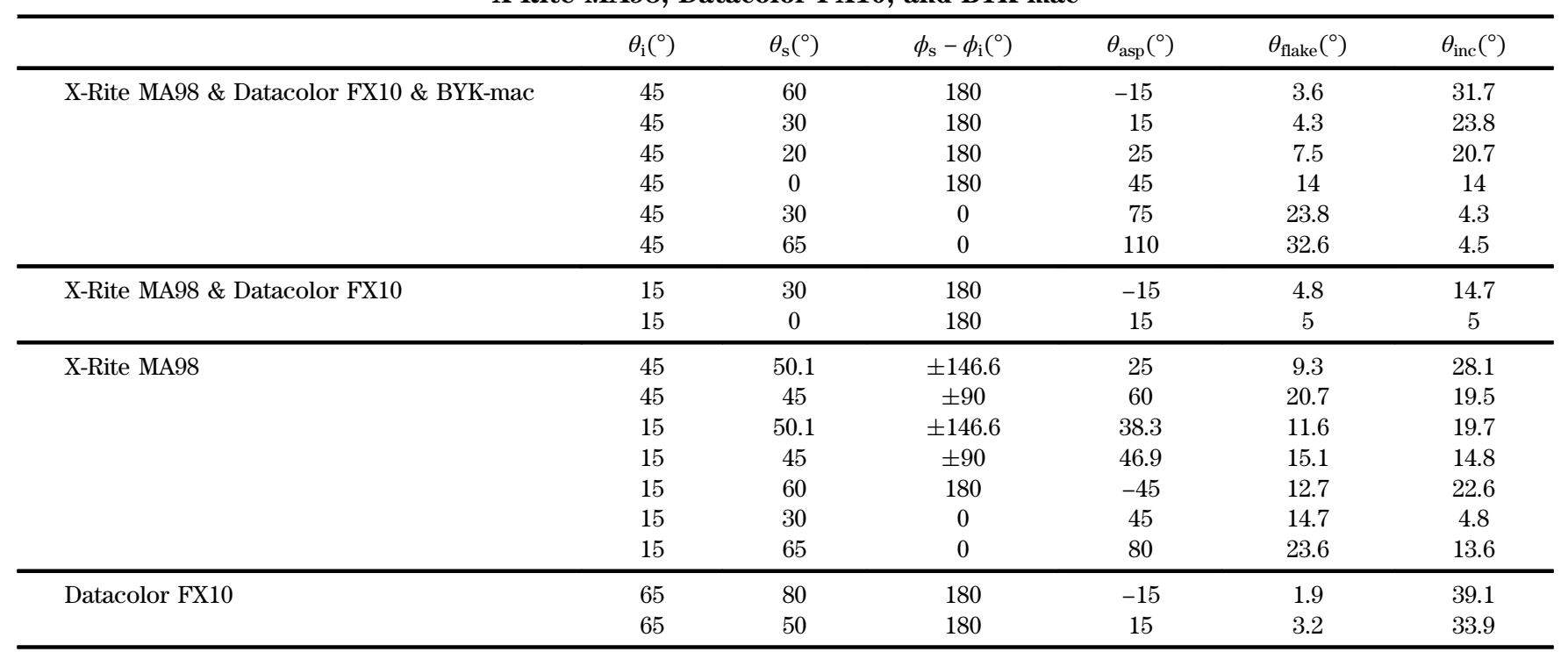




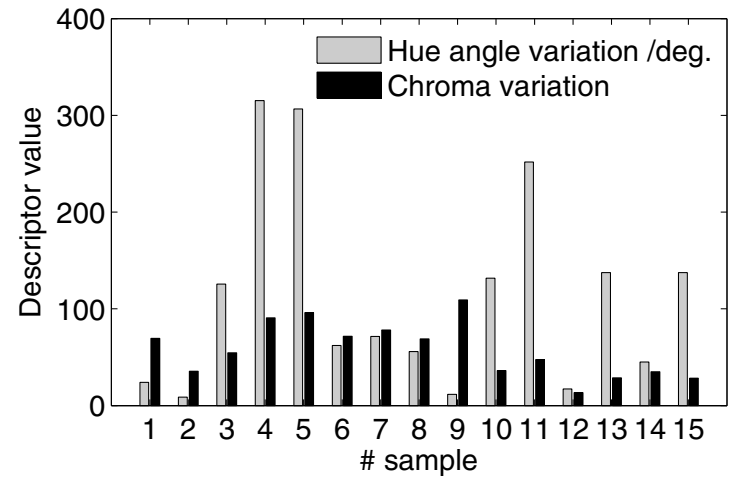

Fig. 2. Total hue angle variation and chroma variation for the 15 studied special-effect coatings. Total hue angle variation was calculated as the highest hue angle difference in the set of geometries with fixed $\theta_{\text {asp }}=10^{\circ}$ and with $\theta_{\mathrm{i}}$ from $0^{\circ}$ to $70^{\circ}$ in steps of $10^{\circ}$. Total chroma angle variation was calculated as the highest hue angle difference in the set of geometries with fixed $\theta_{\mathrm{bi}}=10^{\circ}$ and with $\theta_{\mathrm{i}}$ from $0^{\circ}$ to $70^{\circ}$ in steps of $10^{\circ}$. The bistatic angle $\theta_{\mathrm{bi}}$ is defined as the angular distance between incidence and viewing directions.

FX10. From these instruments, the BYK-mac has the smallest number of geometries. All of them have an illumination polar angle of $\theta_{\mathrm{i}}=45^{\circ}$, and they are included in the two other instruments as well. However, we must mention here that BYK-mac is the only one of these instruments that allows sparkle and graininess to be characterized, which is an important feature for this kind of coatings. X-Rite MA98 and Datacolor FX10 have eight common geometries (six geometries with $\theta_{\mathrm{i}}=45^{\circ}$ and two geometries with $\theta_{\mathrm{i}}=15^{\circ}$ ), and the remaining exclusive geometries will determine the difference in performance. In this regard, X-Rite MA98 has three exclusive in-plane geometries with $\theta_{\mathrm{i}}=15^{\circ}$ and eight exclusive outof-plane geometries. These out-of-plane geometries consist of four pairs of geometries, which are symmetrical with respect to the incident plane (sign \pm in Table 2 ), and almost identical spectral BRDF are expected for these symmetrical geometries. Therefore, X-Rite MA98 has, effectively, seven exclusive geometries, leading to 15 effective geometries in total. On the other hand, Datacolor FX10 has two exclusive in-plane geometries with $\left|\theta_{\text {asp }}\right|=15^{\circ}$ at a higher illumination angle $\left(\theta_{\mathrm{i}}=65^{\circ}\right)$. It has, effectively, 10 different geometries in total.

These geometries are visualized in Fig. 3 by plotting $\theta_{\text {inc }}$ versus $\theta_{\text {flake }}$. The values of these flake-based parameters were calculated by using a refractive index $n=1.5$ for the binder [19]. The X-Rite MA98's plot shows 15 points, representing the 15 effective geometries. The Datacolor FX10's plot shows 10 points. Both instruments have well-distributed geometries within the range of $\theta_{\text {inc }}$ with low $\theta_{\text {flake }}$. Coverage of a large range of values of $\theta_{\text {inc }}$ is important for assessing the color shift due to the interference pigments.

Figure 3 shows that BYK-mac's geometries do give a good range of $\bar{\theta}_{\text {inc }}$ values, although geometries with low values for both $\theta_{\text {inc }}$ and $\theta_{\text {flake }}$ are not present. In the same figure, as another subplot, the nine measurement geometries previously proposed by some of the authors in [18] are also shown.

\section{COLOR ESTIMATION}

Color coordinates are calculated from the spectral BRDF distributions. Therefore, it is necessary to have a procedure to estimate the spectral BRDF for any illumination/viewing geometry from measurements at a reduced set of geometries. This procedure was thoroughly explained in [18], but it will be briefly reviewed here.

\section{A. Review on Spectral BRDF Estimation Procedure} This procedure basically consists of two steps.

Step 1: Determination of a reduced set of spectral distribution functions and coefficients to represent the sample's
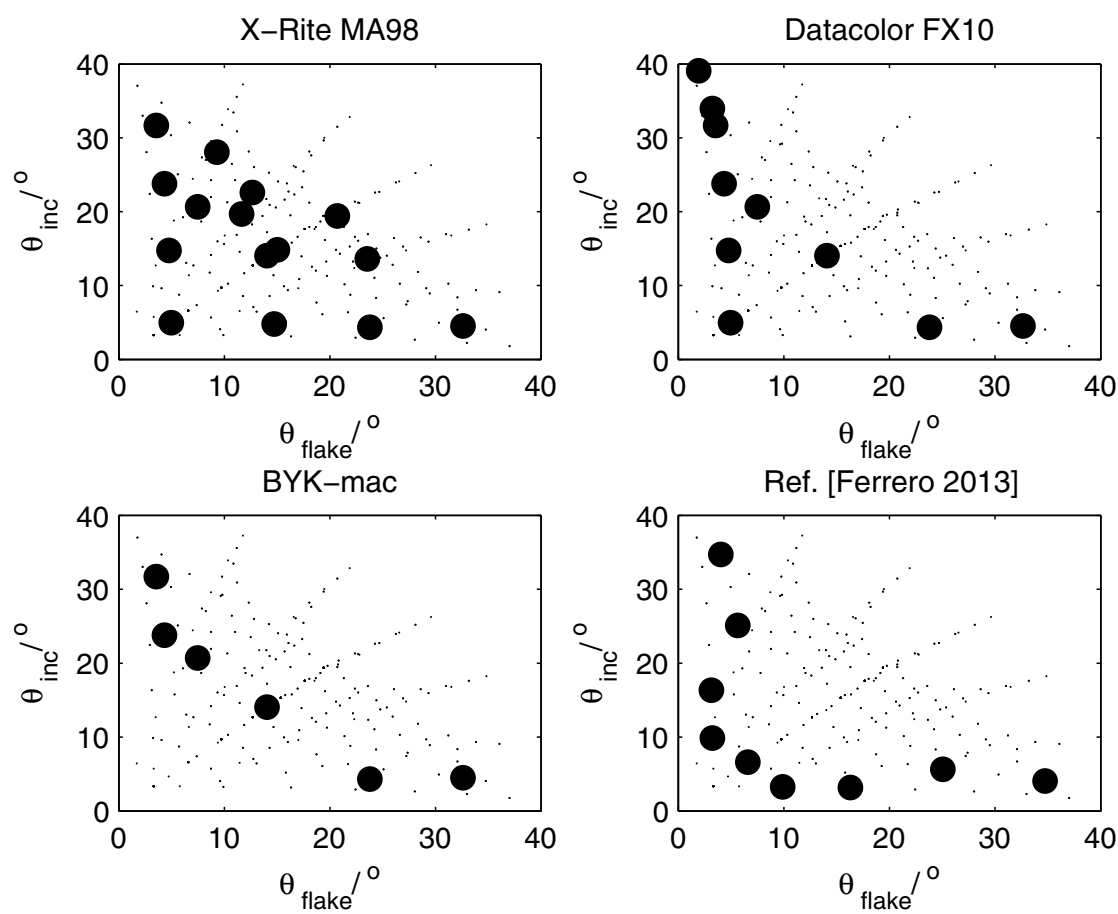


FX10, and BYK-mac) and the nine geometries proposed by some of the authors in [18] (big black circles). The very small marks represent measurement geometries used in this study. 
spectral BRDF. PCA is used to express the spectral BRDF of every special-effect coating as a sum of components, in such a way that spectral and geometrical variables are separated as [18]:

$$
\begin{aligned}
& f_{\mathrm{r}}\left(\theta_{\mathrm{i}}, \phi_{\mathrm{i}} ; \theta_{\mathrm{s}}, \phi_{\mathrm{s}} ; \lambda\right) \\
& \quad=\left\langle f_{\mathrm{r}}\left(\theta_{\mathrm{i}}, \phi_{\mathrm{i}} ; \theta_{\mathrm{s}}, \phi_{\mathrm{s}}\right)\right\rangle_{\lambda}\left[1+\sum_{j=1}^{M} c_{j}\left(\theta_{\mathrm{i}}, \phi_{\mathrm{i}} ; \theta_{\mathrm{s}}, \phi_{\mathrm{s}}\right) H_{j}(\lambda)\right] .
\end{aligned}
$$

This equation is made up of two factors: The spectral average of $f_{\mathrm{r}}\left(\left\langle f_{\mathrm{r}}\left(\theta_{\mathrm{i}}, \phi_{\mathrm{i}} ; \theta_{\mathrm{s}}, \phi_{\mathrm{s}}\right)\right\rangle_{\lambda}\right)$, having just a geometrical dependence; and the sum of the $M+1$ terms shown inside square brackets, where the first one is equal to the unity. Every term nonconstant is the product of two factors: one factor contains the spectral information, $H_{j}(\lambda)$ (having an average value of 0 and a standard deviation of 1 ), and the other factor contains the geometrical information, $c_{j}\left(\theta_{\mathrm{i}}, \phi_{\mathrm{i}} ; \theta_{\mathrm{s}}, \phi_{\mathrm{s}}\right)$, which can be regarded as the weight of the spectral distribution $H_{j}(\lambda)$ at a given geometry.

Step 2: Estimation of angular dependent coefficients for every illumination/viewing geometry. Geometry-dependent variables $\left\langle f_{\mathrm{r}}\right\rangle_{\lambda}$ and $c_{j}$, whose angular dependence has been omitted for the sake of easiness in writing, are interpolated by different functions. A sum of two Gaussians is used to interpolate $\ln \left(\left\langle f_{\mathrm{r}}\right\rangle_{\lambda}\right)$ (designated as $\langle G\rangle$ ), where the independent variable is $\theta_{\text {flake. }}$. The weighting coefficient $c_{1}$, which by definition corresponds to the geometries related with the prevalence of the nonflake scattering, is interpolated by a $2 \mathrm{D}$ function $\left(F_{\text {bcoat }}\right)$ of both $\theta_{\text {flake }}$ and $\theta_{\text {inc }}$ with a decreasing dependence on $\theta_{\text {inc }}$ for higher values of $\theta_{\text {flake }}$ because the higher they are, the less flakes and the less observable is the flake scattering. The other weighting coefficients are interpolated from the expression for the reflection exclusively at the flakes, $D\left(\theta_{\text {flake }}\right) \times f_{\text {r,flake }}\left(\theta_{\text {inc }}, \lambda\right)$, which is a separable function in $\theta_{\text {flake }}$ and $\theta_{\text {inc }}$. On one hand, $D\left(\theta_{\text {flake }}\right)$ is an ensemble function that represents the relative contribution from the special-effect flakes to the total reflection, as function of $\theta_{\text {flake. }}$ This function is usually peaked at $0^{\circ}$ because it is related to the orientation distribution of the flakes. On the other hand, $f_{\text {r,flake }}\left(\theta_{\text {inc }}, \lambda\right)$ represents the spectral reflection of a representative interference pigment, which only depends on $\theta_{\text {inc. }}$. $D\left(\theta_{\text {flake }}\right)$ is estimated by polynomial interpolation (designated as $P_{\mathrm{D}}$ ) of the weighting coefficient $c_{2}$, using only data with low values of $\theta_{\text {inc }}$. Afterward, $f_{\text {r,flake }}\left(\theta_{\text {inc }}, \lambda\right)$ is estimated as the sum of $H_{\mathrm{j}}(\lambda) c_{\mathrm{j}} / P_{\mathrm{D}}\left(\theta_{\text {flake }}\right)$, in which $c_{\mathrm{j}}$ represent all weighting coefficients except $c_{1}$, and interpolated by polynomials $P_{\mathrm{j}}$ with $\theta_{\text {inc }}$ as independent variable, using only those data with low values of $\theta_{\text {flake. }}$.

The interpolated function finally obtained for $f_{\mathrm{r}}$ is written as

$$
\begin{aligned}
f_{\text {r,int }}\left(\theta_{\text {flake }}, \theta_{\text {inc }} ; \lambda\right)= & \mathrm{e}^{\left\langle G\left(\theta_{\text {flake }}\right)\right\rangle}\left[1+F_{\text {bcoat }}\left(\theta_{\text {flake }}, \theta_{\text {inc }}\right) H_{1}(\lambda)\right. \\
& \left.+P_{D}\left(\theta_{\text {flake }}\right) \sum_{j=1}^{M-1} P_{j}\left(\theta_{\text {inc }}\right) H_{j}(\lambda)\right] .
\end{aligned}
$$

\section{B. Color Estimation with Geometries of Commercially Available Instruments}

The spectral BRDF measurements obtained by GEFE and described in Section 2.B were used to calculate the values of the spectral BRDF at the geometries of the commercial instruments (Table 2). They were calculated as the weighted arithmetic mean of the values of the measured BRDF at the four closest measurement geometries to a given commercial geometry, using as weights the inverses of the distances between geometries (defined as the modulus of the difference vector between the unit direction vectors). Specular geometries were excluded from these four closest geometries.

Afterward, the procedure described in Section 3.A was followed for every set of geometries of the listed commercial multiangle spectrophotometers, deriving the function in Eq. (2) for every BRDF set. The performance of this procedure for estimating all the measurements from GEFE, considered as the "true" result, was evaluated by using only the data of the spectral BRDF at the available geometries of each commercial multiangle device as input. This evaluation was done for every sample. To quantify the agreement, CIELAB color difference $\left(\Delta E_{\mathrm{ab}}^{*}\right.$ ) between the color coordinates for standard illuminant
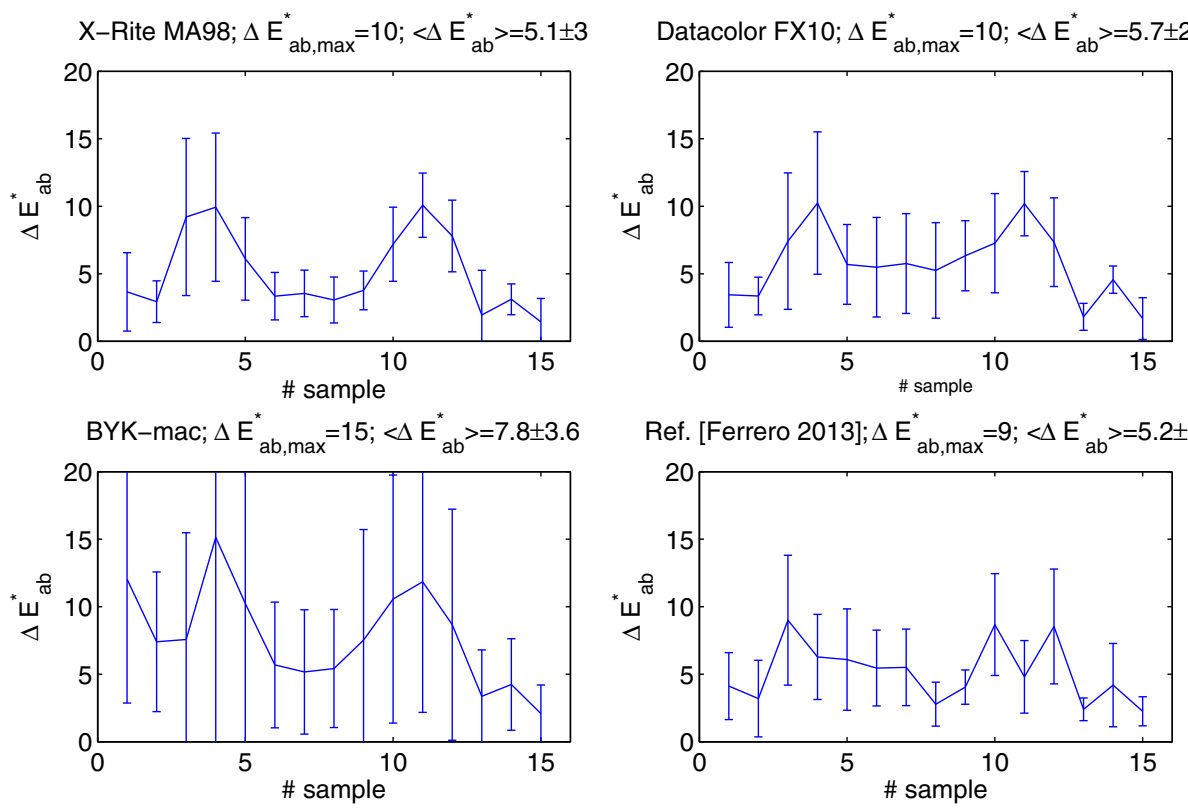

Ref. [Ferrero 2013]; $\Delta \mathrm{E}_{\mathrm{ab}, \max }^{*}=9 ;\left\langle\Delta \mathrm{E}_{\mathrm{ab}}^{*}>=5.2 \pm 2.2\right.$

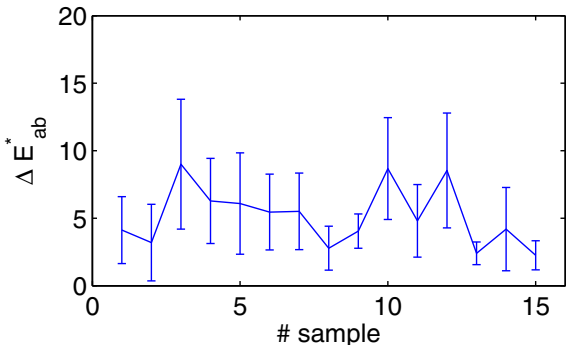

Fig. 4. Average of $\Delta E_{\mathrm{ab}}^{*}$ over all geometries (except specular and retroreflection geometries) for the 15 samples. 

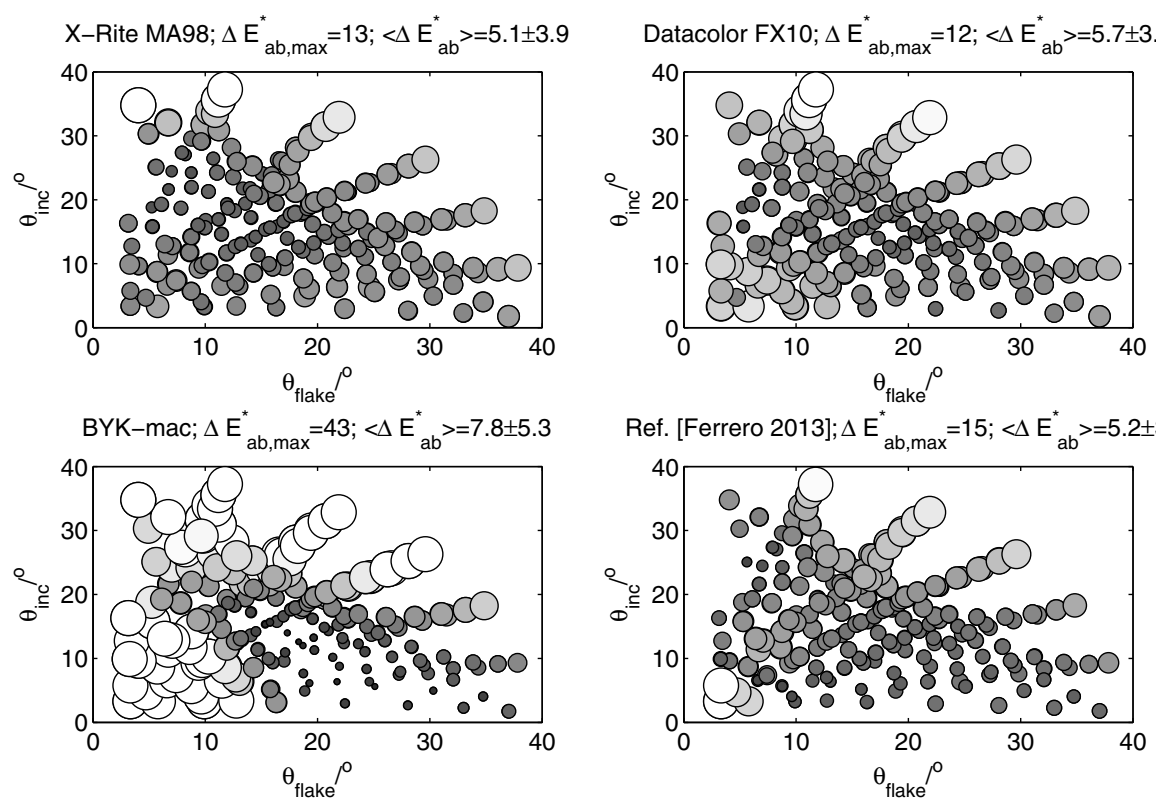

Ref. [Ferrero 2013]; $\Delta \mathrm{E}_{\mathrm{ab}, \max }^{*}=15 ;<\Delta \mathrm{E}_{\mathrm{ab}}^{*}>=5.2 \pm 3.3$

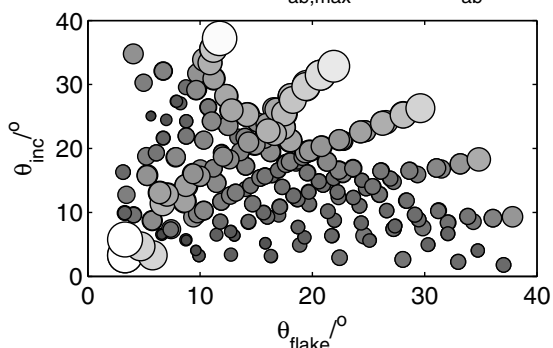

Fig. 5. Bubble chart to show the variation of $\Delta E_{\mathrm{ab}}^{*}$ with the geometry, when color estimation is accomplished from measurements at geometries available in X-Rite MA98, Datacolor FX10, BYK-mac, and at those proposed in [18]. White circles with maximum radii correspond to values of $\Delta E_{\mathrm{ab}}^{*}$ of 10 or more.

D65 and standard observer CIE1964 corresponding to the calculated and measured spectral BRDF values at every measurement geometry was calculated.

The average of $\Delta E_{\mathrm{ab}}^{*}$ over all geometries (except specular and retroreflection ones) for the 15 samples is plotted in Fig. 4 , where the error bars represent the standard deviation of $\Delta E_{\mathrm{ab}}^{*}$. The first three subplots correspond to the commercial instrument sets of geometries, and the fourth to the nine geometries proposed in [18]. The maximum value, the average, and the standard deviation over all samples of the average of $\Delta E_{\mathrm{ab}}^{*}$ over geometries are indicated on the top of each subplot. The latter global parameters show that the performances in estimating special-effect colors when using measurement geometries of X-Rite MA98, Datacolor FX10, and [18]'s geometries are similar $(5.1 \pm 3,5.7 \pm 2.6$, and $5.2 \pm 2.2$, respectively). When using measurement geometries of BYK-mac for estimating BRDF data, the performance is worse $(7.8 \pm 3.6)$ than for the other instruments. This is expected, since the other instruments include more different geometries.

The performance of color estimation varies with the geometry, and this geometry-related performance depends on the selected reduced set of measurement geometries. A bubble chart was chosen to show the variability of $\Delta E_{\mathrm{ab}}^{*}$ with the geometry (Fig. 5), for the studied sets of measurement geometries (X-Rite MA98, Datacolor FX10, BYK-mac, and those proposed in [18]). We represented the position of the assessed measurement geometries in the $\theta_{\text {flake }}-\theta_{\text {inc }}$ coordinates system. The radius of every circle in these charts is linearly related to the value of $\Delta E_{\mathrm{ab}}^{*}$, up to 10 color difference units. Larger differences are represented by the maximum radius. For the sake of clarity, the lightness of every circle also indicates the value of $\Delta E_{\mathrm{ab}}^{*}$, from white (10 or higher) to black (minimum values). In general, the poorest estimation performances (highest circle radii and whitest colors) are located in areas widely separated from the measurement geometries (compare Fig. $\underline{5}$ with Fig. 3 ).
Poorer estimations are naturally obtained in extrapolation areas than in interpolation ones.

We found that the main contributions of partial color differences are due to chroma and hue and not to lightness.

\section{DISCUSSION}

As expected, due to its smaller number of measurement geometries, the color estimation of the proposed procedure is poorer when it is based on BYK-mac geometries than when it is based on geometries of the other instruments, which have more measurement geometries. However, the estimations based on BYK-mac geometries are excellent for geometries with low values of $\theta_{\text {inc }}$ and high values of $\theta_{\text {flake }}$. For those geometries, the procedure surprisingly performs even better when based on BYK-mac geometries than when based on the other instrument's geometries (see Fig. 5). We think this is because the RMSE between the coefficient $c_{1}$ [weighting coefficient related to the scattering in the basecoat, $F_{\text {bcoat }}\left(\theta_{\text {flake }}, \theta_{\text {inc }}\right)$ in Eq. (2)] and its derived function is lower in the case of BYK-mac due to its lower degrees of freedom (less geometries). This better match is important in geometries where the coefficient $c_{1}$ prevails over the other coefficients, as is the case of geometries with high values of $\theta_{\text {flake. }}$. However, keep in mind that, since the set of geometries of BYK-mac is a subset of the geometries of X-Rite MA98 and Datacolor FX10, a better estimation is always attainable when using reflection data from X-Rite MA98 or Datacolor FX10. For instance, we may use only the BYK-mac's subset of geometries to estimate color at low values of $\theta_{\text {inc }}$ and high values of $\theta_{\text {flake }}$ when using X-Rite MA98 or Datacolor FX10.

On the other hand, the evaluation of the color estimation at those geometries with low $\theta_{\text {flake }}$ should be more representative of the capability of the proposed procedure to predict a color shift. In order to assess the estimation performance for those challenging geometries that describe the characteristic color shift of the special-effect coatings, the averages of $\Delta E_{\mathrm{ab}}^{*}$ over geometries for the 15 samples are shown in Fig. 6 , 

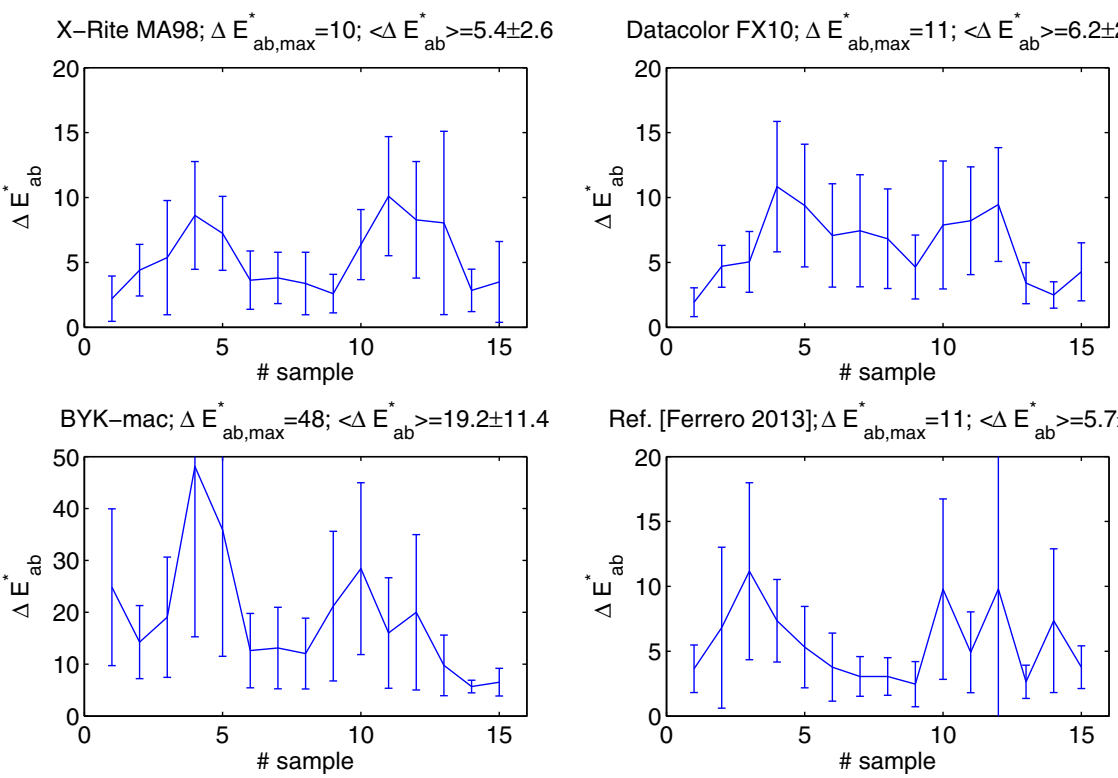

Ref. [Ferrero 2013]; $\Delta \mathrm{E}_{\mathrm{ab}, \max }^{*}=11 ;<\Delta \mathrm{E}_{\mathrm{ab}}^{\star}>=5.7 \pm 2.9$

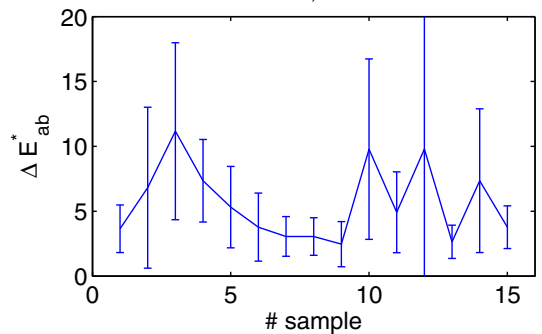

Fig. 6. Average of $\Delta E_{\mathrm{ab}}^{*}$ over geometries with $\theta_{\text {flake }}<7^{\circ}$ (except specular and retroreflection geometries) for the 15 samples.

in the same way as in Fig. 4, but only using the reduced set of the 35 geometries with $\theta_{\text {flake }}<7^{\circ}$. The average over all samples of the average of $\Delta E_{\mathrm{ab}}^{*}$ over geometries $\left(\left\langle\Delta E_{\mathrm{ab}}^{*}\right\rangle\right)$ increased for all set of geometries with respect to the values shown in Fig. $\underline{4}$, but not to the same extent. Whereas in the case of BYK-mac, $\left\langle\Delta E_{\mathrm{ab}}^{*}\right\rangle$ increased from 7.8 to 19.2 ; for the other sets of geometries. the relative increases are significantly smaller (from 5.1 to 5.4 , from 5.7 to 6.2 , and from 5.2 to 5.7 for X-Rite MA98, Datacolor FX10, and for the geometries proposed in [18], respectively).

The values of the average and standard deviation of $\Delta E_{\mathrm{ab}}^{*}$ over geometries, as shown in Fig. 6 , are listed in Table 3 for the 15 studied samples. Samples are sorted from a higher to lower variation of chroma, and the variation of hue angle is also displayed. The best color estimation performance for the five samples with the highest variation of chroma is

Table 3. Average of $\Delta E_{\mathrm{ab}}^{*}$ Over Geometries with $\theta_{\text {flake }}<7^{\circ a}$

\begin{tabular}{|c|c|c|c|c|c|}
\hline $\begin{array}{l}\text { Sample } \\
\text { Number }\end{array}$ & $\begin{array}{c}\text { Chroma } \\
\text { Variation }\end{array}$ & $\begin{array}{c}\text { Hue Angle } \\
\text { Variation } \\
\left({ }^{\circ}\right)\end{array}$ & $\begin{array}{c}\Delta E_{\mathrm{ab}}^{*} \\
\text { X-Rite } \\
\text { MA98 }\end{array}$ & $\begin{array}{c}\Delta E_{\mathrm{ab}}^{*} \\
\text { Datacolor } \\
\text { FX10 }\end{array}$ & $\begin{array}{c}\Delta E_{\mathrm{ab}}^{*} \\
{[\underline{18}]}\end{array}$ \\
\hline 9 & 109.2 & 11.6 & $3.9 \pm 2.5$ & $4.6 \pm 2.5$ & $2.5 \pm 1.7$ \\
\hline 5 & 96.1 & 306.6 & $7.7 \pm 4.0$ & $9.4 \pm 4.7$ & $5.3 \pm 3.1$ \\
\hline 4 & 90.7 & 315.3 & $12.3 \pm 10.7$ & $10.8 \pm 5.0$ & $7.3 \pm 3.2$ \\
\hline 7 & 78.1 & 71.4 & $8.0 \pm 5.5$ & $7.4 \pm 4.3$ & $3.1 \pm 1.5$ \\
\hline 6 & 71.7 & 62.0 & $7.3 \pm 5.1$ & $7.1 \pm 4.0$ & $3.8 \pm 2.6$ \\
\hline 1 & 69.4 & 24.0 & $2.2 \pm 1.4$ & $1.9 \pm 1.1$ & $3.7 \pm 1.8$ \\
\hline 8 & 68.9 & 55.8 & $6.6 \pm 4.6$ & $6.8 \pm 3.8$ & $3.1 \pm 1.4$ \\
\hline 3 & 54.6 & 125.5 & $5.6 \pm 4.8$ & $5.0 \pm 2.3$ & $11.2 \pm 6.8$ \\
\hline 11 & 47.5 & 251.8 & $9.7 \pm 4.6$ & $8.2 \pm 4.2$ & $4.9 \pm 3.1$ \\
\hline 10 & 36.2 & 131.7 & $8.3 \pm 6.2$ & $7.9 \pm 4.9$ & $9.8 \pm 7.0$ \\
\hline 2 & 35.5 & 8.8 & $4.4 \pm 2.1$ & $4.7 \pm 1.6$ & $6.8 \pm 6.2$ \\
\hline 14 & 34.9 & 45.0 & $3.1 \pm 1.4$ & $2.5 \pm 1.0$ & $7.4 \pm 5.5$ \\
\hline 13 & 28.7 & 137.4 & $2.5 \pm 1.9$ & $3.4 \pm 1.6$ & $2.6 \pm 1.3$ \\
\hline 15 & 28.3 & 137.5 & $4.4 \pm 3.6$ & $4.3 \pm 2.2$ & $3.8 \pm 1.6$ \\
\hline 12 & 13.5 & 17.2 & $8.7 \pm 4.7$ & $9.5 \pm 4.4$ & $9.8 \pm 10.9$ \\
\hline
\end{tabular}

${ }^{a}$ Except specular and retroreflection geometries for the 15 samples. Samples are sorted from higher to lower chroma variation. achieved by the set of geometries proposed in [18] and also for all samples with a variation of chroma higher than 54.6 and a variation of hue angle higher than $24^{\circ}$. For this set, we found a small anticorrelation between $\Delta E_{\mathrm{ab}}^{*}$ and the chroma variation (linear correlation $=-0.4$ ). This was unexpected, since it indicates better color estimation for specialeffect coatings with stronger chroma variation. As a matter of fact, samples with the worst color estimation $\left(\Delta E_{\mathrm{ab}}^{*}>7.5\right)$ always have a chroma variation below 55 units. On the contrary, for X-Rite MA98's and Datacolor FX10's geometries, values of $\Delta E_{\mathrm{ab}}^{*}$ higher than 7.5 units are found within almost all the range of chroma variation (13.7-90.7).

We found no correlation with the hue angle variation, when using the geometries proposed in [18]. However, we did find a small positive correlation coefficient of 0.6 for data of X-Rite MA98 and Datacolor FX10. In addition, the geometries in [18] provide better color estimation of the three samples with the highest hue angle variation.

From these results, we can suggest that the proposed procedure, when based on the geometries of X-Rite MA98 or Datacolor FX10, may provide better color estimation for special-effect coatings with relatively small chroma or hue variation with angle. For an instrument based on the measurement geometries proposed in [18], our results suggest that the proposed procedure may give the most accurate color estimations for special-effect coatings with large chroma or hue variation with angle.

The performances of color shift estimations for X-Rite MA98, Datacolor FX10, and the proposed geometries in [18] are visually displayed in $2 \mathrm{D} \mathrm{a}^{*}-\mathrm{b}^{*}$ diagrams in Figs. $7-11$ [experimental data (top left), X-Rite MA98 (top right), Datacolor FX10 (bottom left), and [18] (bottom right)]. In these diagrams, continuous red and discontinuous blue lines represent interference and absorption lines, respectively. An interference line is defined as the locus of calculated color coordinates from measurement geometries with a fixed aspecular angle and an almost constant value of $\theta_{\text {flake, }}$, whereas an absorption line was defined in [22] as the locus of calculated color coordinates from measurement geometries with a fixed 

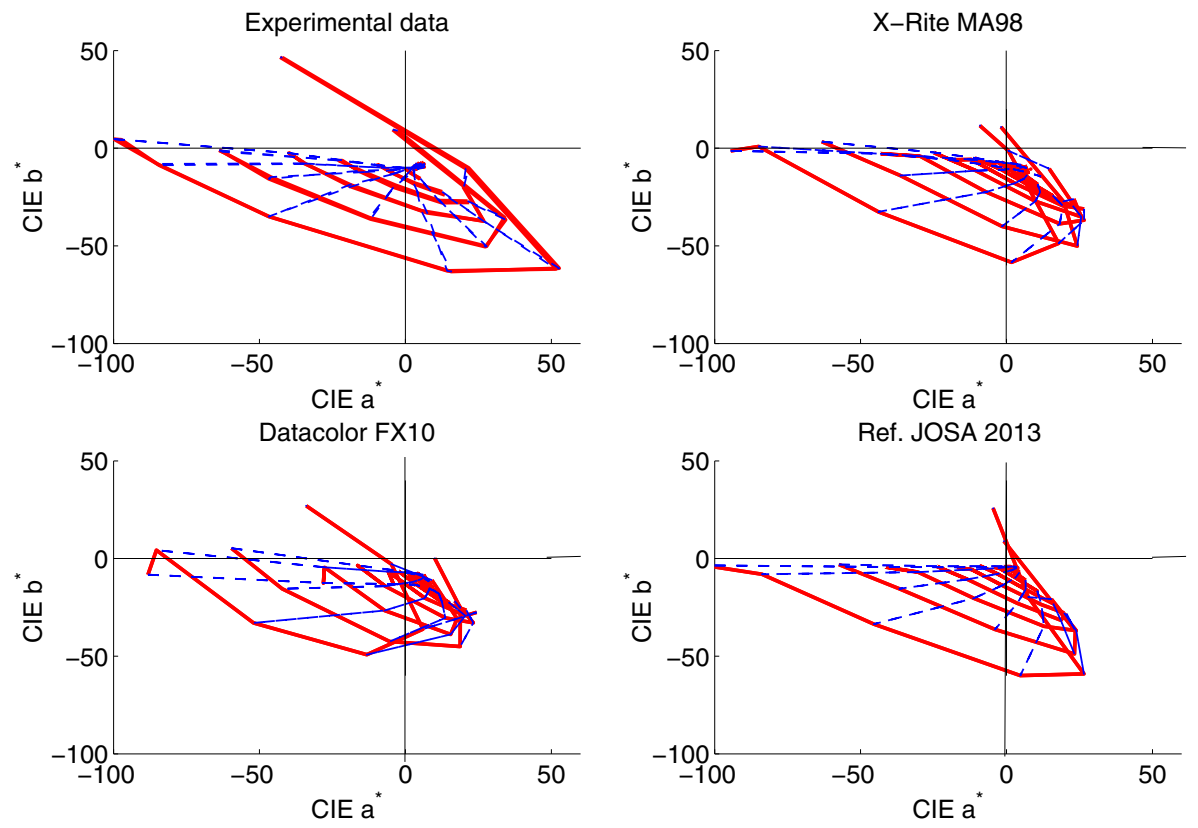

Fig. 7. Estimation of color shift for sample \#4, for which the color estimation is poor for the three sets of geometries. $\Delta E_{\mathrm{ab}}^{*}$ is $8.6,10.8$, and 7.3 for X-Rite MA98, Datacolor FX10, and [18] geometries, respectively.

bistatic angle and an almost constant value of $\theta_{\text {inc }}$. In general, interference lines with higher chroma $\left[C=\left(a^{* 2}+b^{* 2}\right)^{1 / 2}\right]$ correspond to lower values of $\theta_{\text {flake }}$ and better represent the color shift.

The results for a sample where the performance of the color estimation is poor for all three sets of geometries (\#4) are shown in Fig. 7. This is the sample with the largest hue angle variation. $\Delta E_{\mathrm{ab}}^{*}$ is $8.6,10.8$, and 7.3 for the geometries in X-Rite MA98, Datacolor FX10, and [18], respectively. We consider here as acceptable color estimations with $\Delta E_{\mathrm{ab}}^{*}<5$.

The results for a sample with high chroma variation (\#7), for which the color estimation is good for the geometries in [18] $\left(\Delta E_{\mathrm{ab}}^{*}=3.1\right)$ and for X-Rite MA98 $\left(\Delta E_{\mathrm{ab}}^{*}=3.8\right)$, but not so good for Datacolor FX10 $\left(\Delta E_{\mathrm{ab}}^{*}=7.4\right)$, are shown in Fig. 8 .

The color shift in Fig. 9 represents the sample with the largest chroma variation (\#9). Its color estimation is acceptable for the geometries in X-Rite MA98 $\left(\Delta E_{\mathrm{ab}}^{*}=2.6\right)$, Datacolor FX10 $\left(\Delta E_{\mathrm{ab}}^{*}=4.6\right)$, and [18] $\left(\Delta E_{\mathrm{ab}}^{*}=2.5\right)$

The estimation of the color shift of sample \#3 (Fig. 10) is almost acceptable for the geometries in X-Rite MA98 $\left(\Delta E_{\mathrm{ab}}^{*}=5.4\right)$ and Datacolor $\operatorname{FX10}\left(\Delta E_{\mathrm{ab}}^{*}=5\right)$ but not so acceptable for [18] $\left(\Delta E_{\mathrm{ab}}^{*}=11.2\right)$.

The estimation of the color shift shown in Fig. 11 (sample \#15) is good for the geometries in X-Rite MA98 $\left(\Delta \overline{E_{\mathrm{ab}}^{*}}=2.5\right)$, Datacolor FX10 $\left(\Delta E_{\mathrm{ab}}^{*}=4.3\right)$, and [18] $\left(\Delta E_{\mathrm{ab}}^{*}=3.8\right)$.
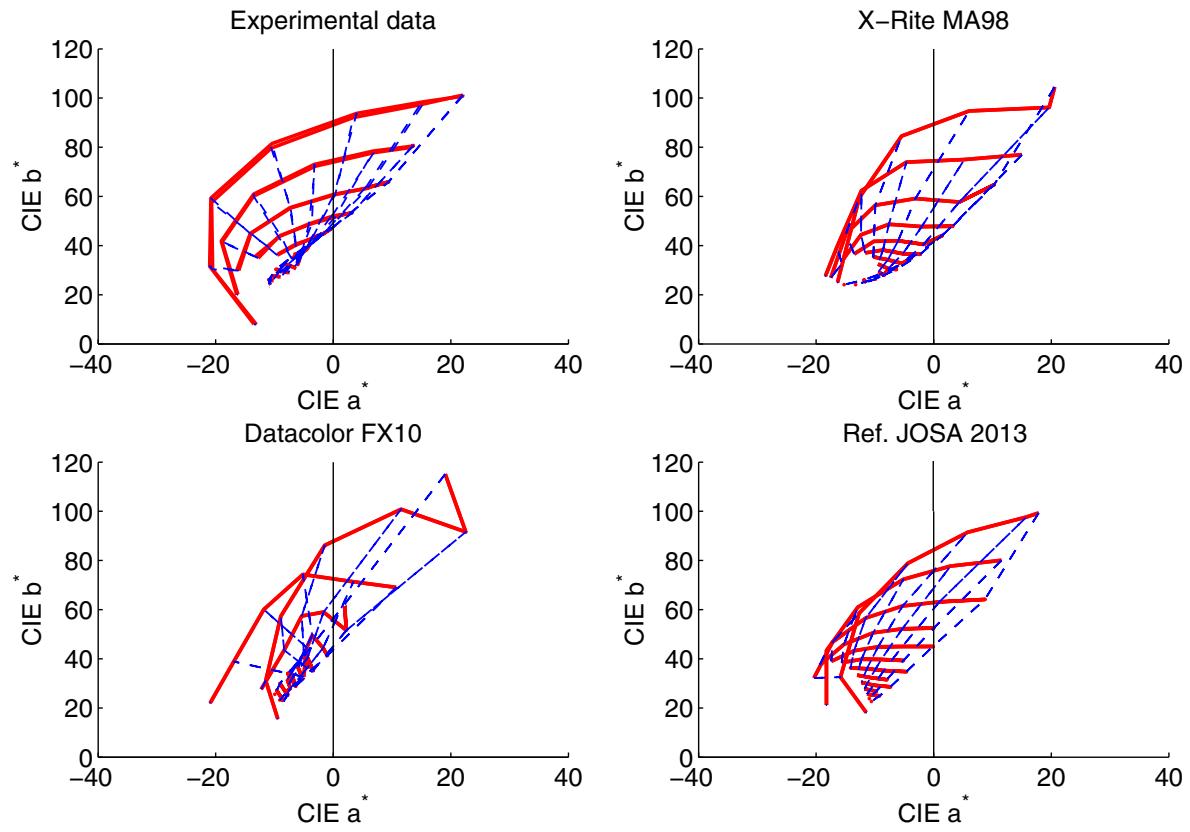

Fig. 8. Estimation of color shift for sample \#7, for which the color estimation is good for the geometries in [18] and X-Rite MA98, but not so good for Datacolor FX10. $\Delta E_{\mathrm{ab}}^{*}$ is 3.8, 7.4, and 3.1 for the geometries in X-Rite MA98, Datacolor FX10, and [18], respectively. 

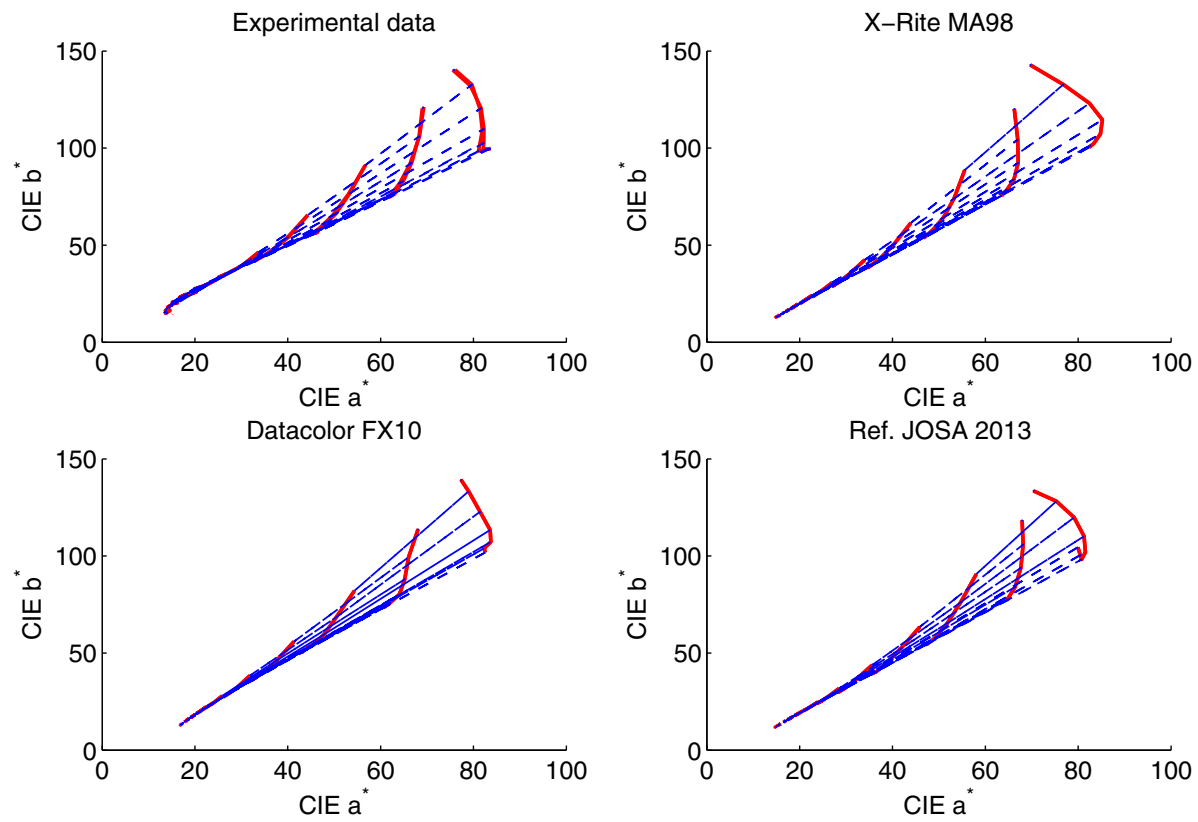

Fig. 9. Estimation of color shift for sample \#9, which presents the highest chroma variation and whose color estimation is acceptable for the geometries in X-Rite MA98, Datacolor FX10, and [18]. $\Delta E_{\mathrm{ab}}^{*}$ is 2.6, 4.6 and 2.5 for the geometries in X-Rite MA98, Datacolor FX10, and [18], respectively.
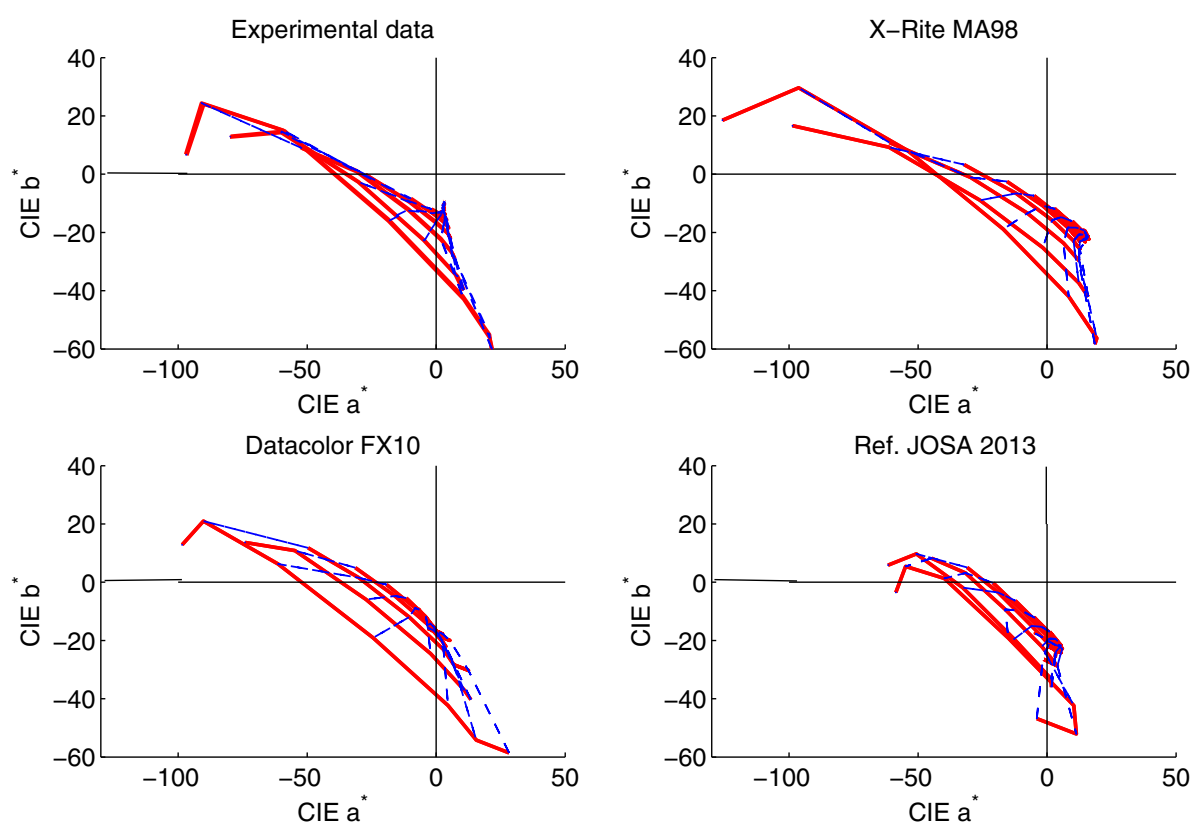

Fig. 10. Estimation of color shift for sample \#3, whose color estimation is almost acceptable for the geometries in X-Rite MA98 and Datacolor FX10 but not so acceptable for [18]. $\Delta E_{\mathrm{ab}}^{*}$ is 5.4, 5, and 11.2 for the geometries in X-Rite MA98, Datacolor FX10, and [18], respectively.

The most noticeable difference between the experimental and the estimated color shifts in Figs. 7-11 is observed in the interference lines with small value of $\theta_{\text {asp }}$. For those geometries, the contribution from reflection at special-effect flakes is more important. In general, the color estimations when basing the proposed procedure on geometries of X-Rite MA98, Datacolor FX10, or [18] are very similar. The good performance of the latter small set of geometries, with only nine geometries may be caused by the fact that these nine geometries were selected aiming to estimate the color shift by having in consideration the PCA-based procedure presented in [18]. In that selection, we made sure to provide adequate sampling for the factors $D\left(\theta_{\text {flake }}\right)$ and $f_{\text {rflake }}\left(\theta_{\text {inc }}, \lambda\right)$ (see Section 3.A), since the product of both factors would represent the special-effect reflectance.

The performance of the proposed procedure was notably worse if the geometries of the BYK-mac are used. This is illustrated in Fig. 12 by the $2 \mathrm{D} a^{*}-b^{*}$ diagrams for samples \#4, \#7, $\# 3$, and \#15. We have compared these diagrams, based on data from BYK-mac geometries, with the corresponding diagrams 

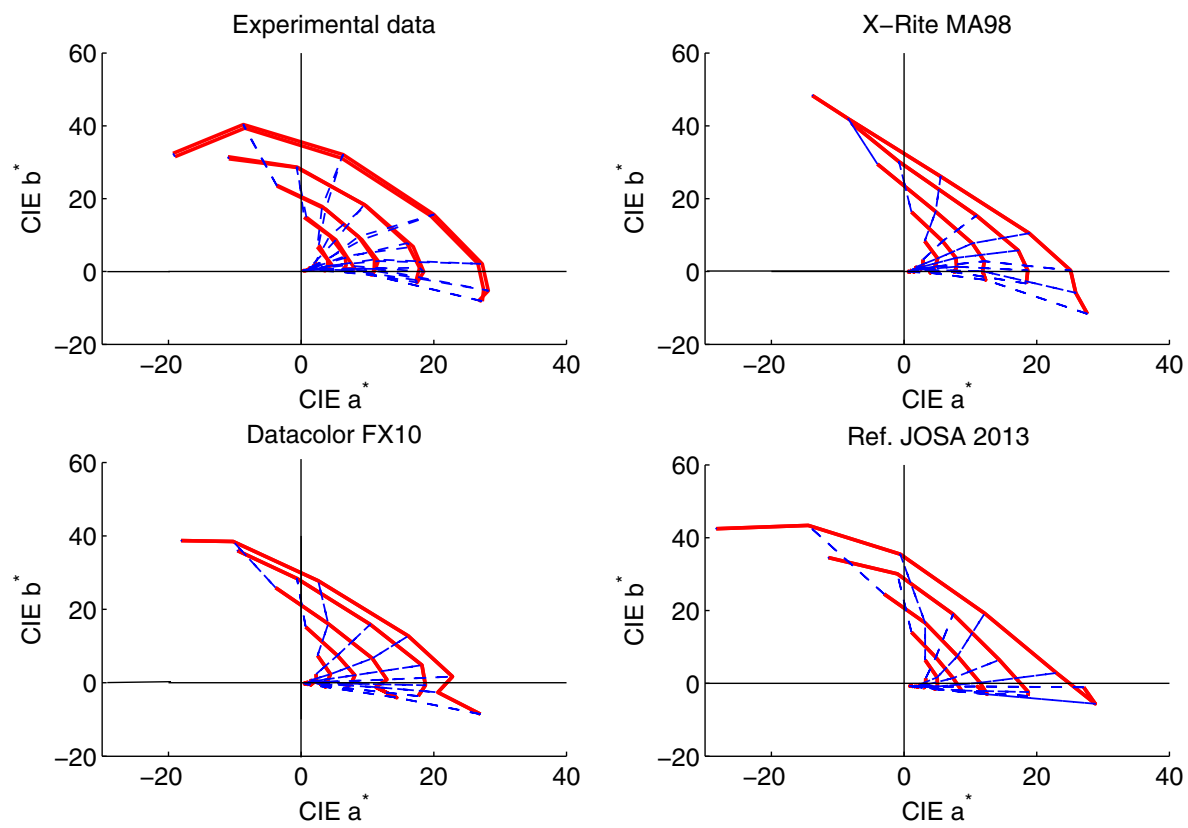

Fig. 11. Estimation of color shift for sample \#15, whose color estimation is good for the geometries in X-Rite MA98, Datacolor FX10, and [18]. $\Delta E_{\mathrm{ab}}^{*}$ is 2.5, 4.3 and 3.8 for the geometries in X-Rite MA98, Datacolor FX10, and [18], respectively.
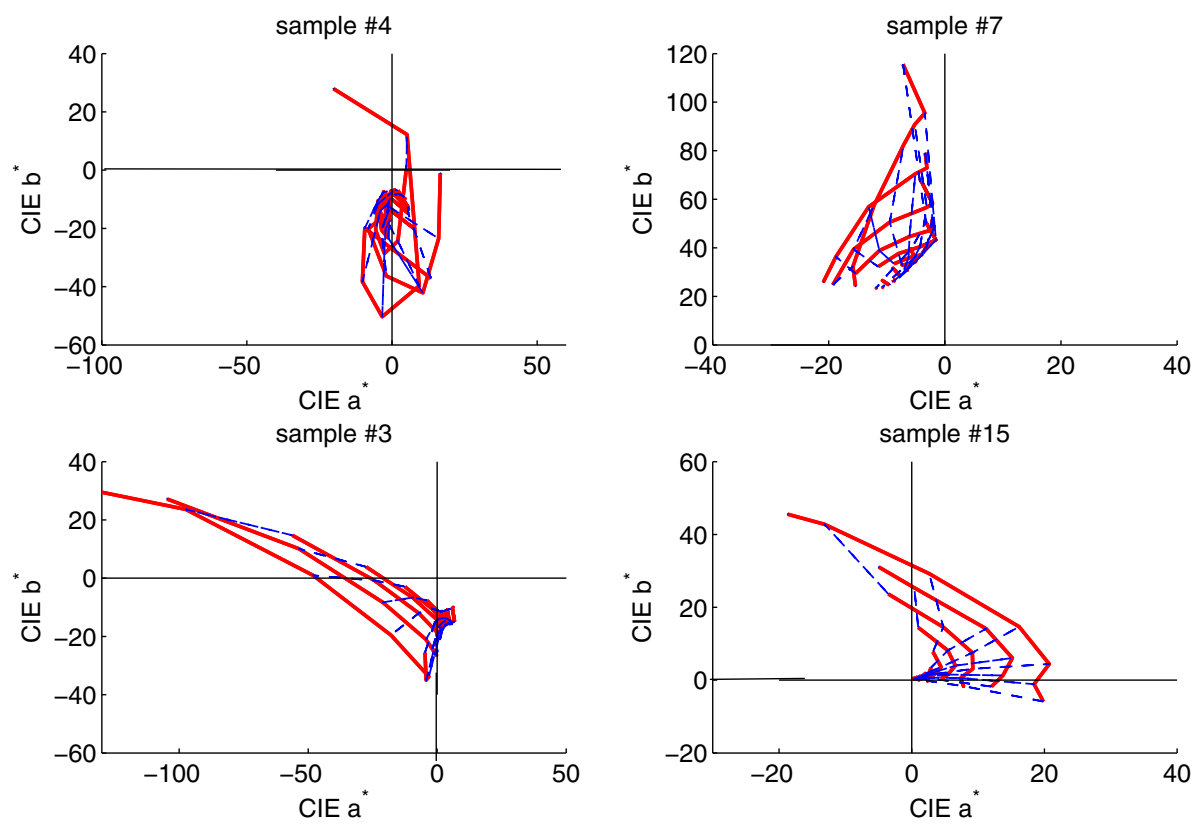

Fig. 12. Estimated color, from the geometries of BYK-mac, for samples \#4 (top left), \#7 (top right), \#3 (bottom left), and \#15 (bottom right), which must be compared with top-left subplots in Figs. $\underline{7}, \underline{8}, \underline{10}$, and $\underline{11} . \Delta E_{\mathrm{ab}}^{*}$ is $48.1,13.1,19.1$, and 6.5 for samples \#4, \#7, \#3, and \#15, respectively.

when utilizing all GEFE data, i.e., with the top-left subplots of Figs. 7, 8, 10, and 11. For sample \#4, which has the highest hue variation, the procedure performs badly. For the other samples, the color estimation is better, but definitively worse than when the other sets of geometries (X-Rite MA98's, Datacolor FX10, or [18]) are used.

\section{CONCLUSIONS}

We have tested the accuracy of a PCA-based procedure presented in [18] to estimate the color shift of special-effect coatings at any measurement geometry, when using BRDF data from a limited set of geometries provided by commercial portable multiangle spectrophotometers: X-Rite MA98, Datacolor FX10, and BYK-mac.

We have measured the spectral BRDF of 15 special-effect samples with the gonio-spectrophotometer GEFE. These samples present a wealth of diversity in terms of angular variation in hue and chroma.

A representative value of $\Delta E_{\mathrm{ab}}^{*}$, as averaged over samples and geometries with small aspecular angles, allowed us to determine the performance of color shift estimation when using the available geometries of each of these instruments. As expected, due to its much smaller number of measurement 
geometries, the procedure showed the worst color estimation $\left(\left\langle\Delta E_{\mathrm{ab}}^{*}=19.2\right\rangle\right)$ when geometries of BYK-mac were used instead of those of X-Rite MA98 and Datacolor FX10, whose color estimations were proven very good for some samples.

This estimation performance was compared with the performance of a hypothetical instrument with only the nine measurement geometries proposed in [18]. The color estimation was comparable with those obtained when using geometries of X-Rite MA98 and Datacolor FX10.

The color estimation procedure evaluated here has great potential for various applications. For example, our results suggest that, for rendering the color appearance of $3 \mathrm{D}$ objects covered with special-effect coatings, the color accuracy obtained with this procedure may be sufficient. This is the case especially if geometries of X-Rite MA98, Datacolor FX10, or those proposed in [18] are used.

\section{ACKNOWLEDGMENTS}

The authors are grateful to EMRP for funding the project "Multidimensional Reflectometry for Industry." The EMRP is jointly funded by the EMRP participating countries within EURAMET and the European Union. This study was also supported by the Spanish Ministry of Economy and Competitiveness under grant no. DPI2011-30090-C02-02 and the European Union. The authors have no interest in the development or marketing of any product mentioned in this study.

\section{REFERENCES}

1. G. Pfaff and P. Reynders, "Angle-dependent optical effects deriving from submicron structures of films and pigments," Chem. Rev. 99, 1963-1982 (1999).

2. F. J. Maile, G. Pfaff, and P. Reynders, "Effect pigments-past, present and future,” Prog. Organic Coat. 54, 150-163 (2005).

3. H. J. Streitberger and K. F. Dössel, Automotive Paints and Coatings (Wiley-VCH, 2008).

4. F. E. Nicodemus, J. C. Richmond, J. J. Hsia, I. W. Ginsberg, and T. Limperis, "Geometrical considerations and nomenclature for reflectance," Natl. Bur. Stand. Monogr. 160 (1977).

5. A. M. Rabal, A. Ferrero, J. Campos, J. L. Fontecha, A. Pons, A. M. Rubiño, and A. Corróns, "Automatic gonio-spectrophotometer for the absolute measurement of the spectral BRDF at in \& out-of-plane and retroreflection geometries," Metrologia 49, 213-223 (2012).

6. G. Obein, R. Bousquet, and M. E. Nadal, "New NIST reference goniospectrometer," Proc. SPIE 5880, 58800T (2005).

7. T. A. Germer and C. C. Asmail, "Goniometric optical scatter instrument for out-of-plane ellipsometry measurements," Rev. Sci. Instrum. 70, 3688-3695 (1999)
8. D. Hünerhoff, U. Grusemann, and A. Höpe, "New robot-based gonioreflectometer for measuring spectral diffuse reflection," Metrologia 43, S11-S16 (2006).

9. F. B. Leloup, S. Forment, P. Dutré, M. R. Pointer, and P. Hanselaer, "Design of an instrument for measuring the spectral bidirectional scatter distribution function," Appl. Opt. 47, 54545467 (2008).

10. R. Baribeau, W. S. Neil, and É. Côté, "Development of a robotbased gonioreflectometer for spectral BRDF measurement," J. Mod. Opt. 56, 1497-1503 (2009).

11. S. Nevas, F. Manoocheri, and E. Ikonen, "Gonioreflectometer for measuring spectral diffuse reflectance," Appl. Opt. 43, 63916399 (2004).

12. N. Matsapey, J. Faucheu, M. Flury, and D. Delafosse, "Design of a gonio-spectrophotometer for optical characterization of gonioapparent materials,” Meas. Sci. Technol. 24, 065901 (2013).

13. S. Ouarets, G. Ged, A. Razet, and G. Obein, "A new gonioreflectometer for the measurement of the bidirectional reflectance distribution function (BRDF) at LNE-CNAM," in Proceedings of CIE 2012 Lighting Quality and Energy Efficiency (2012), pp. $687-691$

14. H. J. Patrick, C. J. Zarobila, and T. A. Germer, "The NIST robotic optical scatter instrument (ROSI) and its application to BRDF measurements of diffuse reflectance standards for remote sensing," Proc. SPIE 8866, 886615 (2013).

15. A. Takagi, S. Sato, and G. Baba, "Prediction of spectral reflectance factor distribution of color-shift paint finishes," Color Res. Appl. 32, 378-387 (2007).

16. "Standard practice for multiangle color measurement of interference pigments," ASTM E 2539-08 (American Society for the Testing of Materials, West Conshohocken, Pennsylvania 2009.

17. M. E. Nadal and E. A. Early, "Color measurements for pearlescent coatings," Color Res. Appl. 29, 38-42 (2004).

18. A. Ferrero, A. M. Rabal, J. Campos, F. Martínez-Verdú, E. Chorro, E. Perales, A. Pons, and M. L. Hernanz, "Spectral BRDF-based determination of proper measurement geometries to characterize color shift of special effect coatings," J. Opt. Soc. Am. A 30, 206-214 (2013).

19. E. Kirchner and W. Cramer, "Making sense of measurement geometries for multi-angle spectrophotometers," Color Res. Appl. 37, 186-198 (2012).

20. E. Kirchner and A. Ferrero, "Isochromatic lines as extension of the Helmholtz reciprocity principle for effect paints," J. Opt. Soc. Am. A, 31, 1861-1867 (2014).

21. A. Ferrero, B. Bernad, J. Campos, F. M. Martínez-Verdú, E. Perales, I. van der Lans, and E. Kirchner, "Towards a better understanding of the color shift of effect coatings by densely sampled spectral BRDF measurement," Proc. SPIE 9018, 90180K (2014).

22. A. Ferrero, E. Perales, A. M. Rabal, J. Campos, F. M. MartínezVerdú, E. Chorro, and A. Pons, "Color representation and interpretation of special effect coatings," J. Opt. Soc. Am. A 31, 436-447 (2014). 